\title{
Realizar una apendicectomía dentro de las 24 horas de comenzado el cuadro de apendicitis no modificaría los resultados
}

Performing apendicectomy in the first $\mathbf{2 4}$ hours of the clinical presentation does not seem to modify outcomes.

Abou-Nukta F y col. Arch Surg. 2006; 141:504-6

\section{Objetivo}

Determinar si la espera de 12 a 24 horas en la realización de una apendicectomía, con el fin de minimizar la alteración de la programación de quirófano y el número de cirugías nocturnas, afecta negativamente a los pacientes con diagnóstico de apendicitis aguda.

\section{Diseño}

Cohorte retrospectiva* por revisión de historias clínicas.

\section{Lugar}

Connecticut, Estados Unidos.

\section{Pacientes}

309 adultos de 18 a 90 años con diagnóstico de apendicitis aguda confirmada por histopatológica. Criterios de exclusión: apendicectomías incidentales y más de 24 horas de evolución del cuadro clínico.

\section{Medición de resultados principales}

Días de internación, tiempo quirúrgico, tasa de perforación y complicaciones.

\section{Resultados principales}

Los pacientes fueron divididos en dos grupos: 1) cirugía "temprana" o dentro de las primeras 12 horas luego del diagnóstico incluyendo horarios nocturnos $(n=233) ; 2)$ cirugía "tardía" o entre 12 y 24 horas luego del diagnóstico y siempre durante horas diurnas $(n=6)$. No hubo diferencias significativas en los resultados. Ver tabla 1.

Tabla 1: principales resultados.

\begin{tabular}{|c|c|c|c|c|}
\hline & $\begin{array}{l}\text { Cirugía } \\
\text { temprana }(\mathrm{n}=233)\end{array}$ & $\begin{array}{c}\text { Cirugía } \\
\text { tardia }(n=76)\end{array}$ & \\
\hline \multicolumn{2}{|c|}{ Tiempo a la cirugía } & $6,7+/-2,7 \mathrm{hs}$ & $16,7+/-3,6 \mathrm{hs}$ & \multirow{7}{*}{$\begin{array}{l}\text { Diferencias no } \\
\text { significativas }\end{array}$} \\
\hline \multicolumn{2}{|c|}{ Duración de la internación } & $2,5+/-2,3$ hs & $2,9+/-1,8 \mathrm{hs}$ & \\
\hline \multicolumn{2}{|c|}{ Apendicitis avanzada } & $75(32 \%)$ & $28(37 \%)$ & \\
\hline \multirow{2}{*}{$\begin{array}{l}\text { Duración } \\
\text { de la cirugía }\end{array}$} & Laparoscopía & $81+/-31 \mathrm{~min}$. & $81,5+/-31 \mathrm{~min}$. & \\
\hline & Cirugía convencional & 77 +/- $31 \mathrm{~min}$. & $86+/-33 \mathrm{~min}$. & \\
\hline \multirow[t]{2}{*}{ Complicaciones } & Absceso & $6(2 \%)$ & $1(1 \%)$ & \\
\hline & Infección de la herida & $2(<1 \%)$ & $1(1 \%)$ & \\
\hline
\end{tabular}

\section{Conclusiones}

Una aproximación al tratamiento de la apendicitis que incluya la administración precoz de antibióticos endovenosos y la hidratación parenteral seguida de una apendicectomía programada no aumenta la tasa de complicaciones ni los tiempos de internación o de cirugía. Este enfoque permite mantener la agenda de quirófano dando lugar a las eventuales emergencias, además de maximizar el uso de recursos humanos y materiales.

Palabras clave: apendicectomía, cirugía tardía, cohorte retrospectiva. Key words: apendicectomy, late surgery, retrospective cohort. Fuente de financiamiento: No referida.

\section{Comentario}

La apendicectomía es la cirugía abdominal más común en la emergencia y aproximadamente $8 \%$ de las personas sufrirá apendicitis en algún momento de su vida'. Además siguen observándose casos de diagnóstico muy tardío, con sepsis, abscesos hepáticos, falla multiorgánica e incluso óbito. Su diagnóstico es difícil, especialmente en los menores de cinco $a_{n}{ }^{2}$, ya que su cuadro clínico se confunde con dolores habituales, gastroenterocolitis y diarreas, siendo de vital importancia la evaluación precoz y la reevaluación por el especialista, la hidratación y el ayuno.

Si bien el estudio observacional que comentamos no mostró diferencias de resultado entre la resolución en agudo y una pequeña espera para acomodar los horarios de quirófano y evitar cirugías nocturnas, destacamos que no aleatorizó los grupos, por lo que estos que podrían no ser comparables desde el inicio. Como dato a favor vale mencionar que fueron equivalentes en relación a su temperatura y recuento de leucocitos al ingreso (posibles variables confundidoras conocidas) y que los autores reconocen que quizás el estudio no contó con el poder suficiente para detectar una diferencia entre los grupos en relación a la tasa de complicaciones.

\section{Conclusiones del comentador}

En nuestro medio podrían tomarse medidas similares en algunos centros. Sin embargo, es conocida la asimetría existente entre la medicina privada y la pública en cuanto a la accesibilidad de la población, lo que conlleva a consultas en estadios más avanzados y automedicación más frecuente. Una vez tomada la decisión quirúrgica, con el paciente en ayunas, hidratado y medicado con antibióticos (eventualmente con analgésicos y antiácidos) es más importante la estabilización preoperatoria que la urgencia por ir a quirófano.

Ver glosario*

Marcelo Boer [ Servicio de Cirugía Pediátrica Hospital Italiano Buenos Aires. ]

Laura Bortolin [ Estudiante de la Escuela de Medicina del Instituto Universitario Hospital Italiano Buenos Aires. ]

Recibido el 05/02/2007 y aceptado el 12/04/2007

Boer M, Bortolin L. Realizar una apendicectomía dentro de las 24 horas de comenzado el cuadro de apendicitis no modificaría los resultados. Evid. actual. práct. ambul; 10(2):40, mar-abr.2007. Comentado de: Abou-Nukta F, Bakhos C, Arroyo K y col. Effects of delaying appendectomy for acute appendicitis for 12 to 24 hours. Arch Surg. 2006 May; 141(5):504-6. PMID: 16702523.

Referencia

1. Addiss DG, Shaffer N, Fowler BS, et al. The epidemiology of appendicitis and appendectomy in the United States. Am J Epidemiol 1990; $132: 910-925$

2. Kwok MY, Kim MK, Gorelick MH. Evidence-based approach to the diagnosis of appendicitis in children. Pediatr Emerg Care. 2004 Oct;20 (10):690-8; quiz 699-701.Omundsen M, Dennett E. 